\title{
Impact of nitric oxide on proline and putrescine biosynthesis in Chlamydomonas via transcriptional regulation
}

\author{
Z. ZALUTSKAYA ${ }^{1}$, V. DERKACH ${ }^{1}$, R. PUZANSKIY², and E. ERMILOVA ${ }^{1 *}$ \\ Biological Faculty, Saint-Petersburg State University, Saint-Petersburg 199034, Russia ${ }^{1}$ \\ Komarov Botanical Institute of the Russian Academy of Sciences, Saint-Petersburg 197376, Russia ${ }^{2}$
}

\begin{abstract}
Nitric oxide plays an important role in regulating adaption of the model alga Chlamydomonas reinhardtii to various environmental stresses. One response to abiotic stresses is the accumulation of protective molecules such as proline and putrescine. The NO treatment led to a significant accumulation of proline in cells. Quantitative real-time expression analysis of proline metabolic genes in NO-treated cells showed a prolonged upregulation of the gene encoding $\gamma$-glutamyl kinase 1 (GGK1) in the glutamate biosynthetic pathway. Furthermore, truncated hemoglobin 2 (THB2)-underexpressing strains with an enhanced endogenous NO demonstrated a higher proline content and GGK1 mRNA abundances than the wild type. In contrast, transcription of the gene encoding ornithine $\delta$-aminotransferase in the ornithine pathway of proline biosynthesis decreased after treatment with NO. This suggests the predominance of the glutamate pathway over the ornithine pathway. We also found that the expression of the proline dehydrogenase gene encoding a key enzyme in proline catabolism was downregulated in NO-treated cells. Chlamydomonas reinhardtii exposed to exogenous NO also showed an increased ornithine decarboxylase 2 mRNA and putrescine content. Our findings indicate a clear link between changes in NO application and proline and putrescine content via transcriptional regulation of respective enzymes.
\end{abstract}

Additional key words: glutamyl kinase, ornithine decarboxylase, polyamines, truncated hemoglobins.

\section{Introduction}

Nitric oxide is a gaseous secondary messenger that has evolved as an important and ubiquitous signal molecule in plants (Wendehenne and Hancock 2011, Domingos et al. 2015). In higher plants, NO has been implicated in many physiological processes, such as development, reproduction, resistance to various abiotic and biotic stresses (Corpas et al. 2011, Mur et al. 2013, Domingos et al. 2015, Corpas and Barroso 2015, Farnese et al. 2016). In the model green alga Chlamydomonas reinhardtii, NO is also involved in a number of cell functions including macro-nutrient stress responses, hypoxia, and salt stress (Hemschemeier et al. 2013, Wei et al. 2014, Chen et al. 2016, Minaeva et al. 2017, De Mia et al. 2019, Filina et al. 2019). Moreover, as in higher plants (Milani et al. 2003), in $C$. reinhardtii, truncated hemoglobins (e.g. THB1 and THB2) have also been implicated in NO scavenging to protect cells from the accumulation of the radical molecule (Sanz-Luque et al. 2015, Minaeva et al. 2017, Filina et al. 2019). Remarkably, despite this plethora of NO effects, its role in the potential control of the synthesis of protective organic molecules remains largely unknown.

Among many protective molecules, proline is the most widely accumulated compound in higher plants under stress conditions and this amino acid has multifunctional roles (Verbruggen and Hermans 2008, Szabados and Savouré 2010, Sharma et al. 2011, Dar et al. 2016, Signorelli 2016, Chun et al. 2018). In C. reinhardtii, proline has been shown to prime cells against salt stress (Mastrobuoni et al. 2012).

As in many other plants (Szepesi and Szőllösi 2018), proline is synthesized by $C$. reinhardtii from either glutamate or ornithine (Fig. 1). In higher plants, the key enzyme in the biosynthesis of proline from glutamate is a bifunctional $\Delta^{1}$-pyrroline 5-carboxylate synthase (P5CS), which catalyzes the conversion of glutamate to $\gamma$-glutamyl phosphate (Verbruggen and Hermans 2008). However, in $C$. reinhardtii, two separate enzymes, $\gamma$-glutamyl kinase and $\gamma$-glutamyl phosphate reductase, metabolize glutamate (Miyoshi et al. 2011). This is similar to the first two steps of the glutamate pathway in a number of bacteria that use $\gamma$-glutamyl kinase and $\gamma$-glutamyl phosphate reductase

Submitted 23 March 2020, last revision 18 June 2020, accepted 19 June 2020. NO Special Issue

Abbreviations: DEA-NONOate - 2-(N,N diethylamino)-diazenolate-2-oxide sodium salt; GGK1 - glutamyl kinase 1; ODC2 - ornithine decarboxylase 2; OTA1 - ornithine $\delta$-aminotransferase 1; P5C - pyrroline-5-carboxylate; TAP - tris-acetate-phosphate; THB - truncated hemoglobin; WT - wild type.

Acknowledgements: This research was funded by the Russian Science Foundation (No. 16-14-10004).

* Corresponding author; e-mail: e.ermilova@spbu.ru 
(Commichau et al. 2008, Fichman et al. 2015). Notably, in some bacteria, osmostress-adaptive synthesis of proline is controlled at the level of transcription (Hoffmann and Bremer 2016). Ornithine is the alternate precursor, which can be transaminated to pyrroline-5-carboxylate (P5C) by ornithine- $\gamma$-aminotransferase (OAT) (Fig. 1). Proline accumulation in salt-stressed $C$. reinhardtii raises the question about potential stress-inducible genes encoding enzymes of glutamate and/or ornithine pathways. This conundrum has yet to be addressed experimentally.

Apart from neutrally charged protective molecules (such as proline), polyamines are also accumulated in response to stress in a variety of plants (Sengupta et al. 2016). The most commonly occurring polyamine is putrescine. In higher plants, putrescine is formed primarily via the decarboxylation of arginine by arginine decarboxylase and subsequent degradation of the generated agmatine (Slocum 1991). In C. reinhardtii, putrescine synthesis is controlled by ornithine decarboxylase (ODC) (Fig. 1; Voigt et al. 2000). Importantly, putrescine is accumulated in salt-treated C. reinhardtii cells (Mastrobuoni et al. 2012). Moreover, NO plays a role in adaptive response of the alga to salt stress (Chen et al. 2016). We hypothesize the potential relationship(s) between changes in NO content and proline and/or putrescine content via transcriptional regulation of enzyme(s).

The aims of this study were to investigate the impact of $\mathrm{NO}$ on transcriptional regulation of proline and putrescine biosynthesis and to establish a relationship between the proline and putrescine accumulation, the expression of genes of their biosynthetic pathways and NO content.

\section{Materials and methods}

Strains and experimental conditions: The following Chlamydomonas reinhardtii strains were used: wildtype cw15-325 ( $m t+, c w 15$, arg7), which was kindly provided by Dr. M. Schroda (University of Kaiserslautern, Germany) and transformants with reduced THB2 obtained from cw15-325 amiTHB2-7 ( $m++, c w 15)$, amiTHB2-17 $(m t+, c w 15)$ and amiTHB2-22 $(m t+, c w 15)$ (Filina et al. 2019). Cells were grown in tris-acetate-phosphate (TAP) medium (http://www.chlamy.org/TAP.html) under a continuous irradiance of $20 \mu \mathrm{mol}$ (photons) $\mathrm{m}^{-2} \mathrm{~s}^{-1}$ and a temperature of $22{ }^{\circ} \mathrm{C}$ with constant orbital agitation at 90 rpm (Fig. 1 Suppl.). The TAP medium was supplemented with $100 \mathrm{mg} \mathrm{dm}^{-3}$ arginine when required. The 2-(N,N diethylamino)-diazenolate-2-oxide sodium salt (DEANONOate, Sigma-Aldrich, St. Louis, USA) was added to the medium to a final concentration of $50 \mu \mathrm{M}$ or $100 \mu \mathrm{M}$ and then cells were incubated for $0.5,1,2$, or $4 \mathrm{~h}$. At each harvesting times, cells were washed in TAP medium and analyzed as described below. For the salt stress analysis, the cells grown in TAP medium were collected at the midexponential phase of growth by centrifugation $(4000 \mathrm{~g}$, $5 \mathrm{~min}$ ), and then incubated in TAP with addition of $\mathrm{NaCl}$ to a final concentration of $150 \mathrm{mM}$. For all measurements, samples were taken from cultures $1,2,4$, and $6 \mathrm{~h}$ after $\mathrm{NaCl}$ supply. To induce hypoxia conditions, cultures were grown at $22{ }^{\circ} \mathrm{C}$ to $\log$ phase $\left(5-7 \times 10^{6}\right.$ cells $\left.\mathrm{cm}^{-3}\right)$ in $1-\mathrm{dm}^{3}$ flasks. Then, for experiments, $50 \mathrm{~cm}^{3}$ aliquots of cells were transferred into sealed $50-\mathrm{cm}^{3}$ square glass bottles as described (Ostroukhova et al. 2017). The flasks were subsequently incubated in the dark, allowing a gradual removal of dissolved oxygen by respiratory activity. Dissolved oxygen was assayed using a Clarktype electrode to ensure that hypoxic conditions were achieved. In each experiment, the number of cells was measured employing a counting chamber and the viable cells were estimated microscopically with use of $0.0125 \%$ (v/v) methylene blue (DIA-M, Moscow, Russia). Stained (non-viable) and unstained (viable) cells were observed and counted. Four hundred cells from each sample were examined for three biological replicates.

Measurement of chlorophyll, proline, and putrescine content: Determination and calculations of total chlorophyll were performed as previously described (Minaeva et al. 2017). The proline content was measured at $520 \mathrm{~nm}$ as described (Bates et al. 1973). For putrescine content analysis, $10^{8}$ cells were collected by gentle centrifugation at $4000 \mathrm{~g}$ and each pellet was immediately frozen with liquid nitrogen. Metabolites were extracted and derivatized as was previously reported (Puzanskiy et al. 2018). gasm chromatography - mass spectrometry analysis was performed at Agilent 5860 chromatograph (Agilent Technologies, Santa Clara, CA, USA) with Agilent $5975 \mathrm{~S}$ mass selective detector. Separation was performed on a $J \& W H P-5 m s$ capillary column. Helium flow rate was $1 \mathrm{~cm}^{3} \mathrm{~min}^{-1}$. Inlet temperature was $250^{\circ} \mathrm{C}$ at splitless mode. The temperature conditions of the column thermostat were the following: initial temperature of $70{ }^{\circ} \mathrm{C}$, increased by $6{ }^{\circ} \mathrm{C}$ per min up to $325^{\circ} \mathrm{C}$. Electron impact ionization was performed at $70 \mathrm{~V}$ and an ion source temperature of $230^{\circ} \mathrm{C}$. To verify putrescine peaks, the standard of it was analyzed (Sigma-Aldrich). Arbitrary quantification was made as normalization of metabolite peak area by internal standard (tricosane) peak area. The analysis of the GC-MS data was processed using the PARADISE program (Johnsen et al. 2017) in association with NIST MS search program and mass-spectrometer library NIST2010 (National Institute of Standards and Technology, Gaithersburg, MD, USA). Statistical processing was performed in the language environment $R$ 3.5.2 "Eggshell Igloo" ( $R$ Core Team 2020). An additional post-hoc test for mean comparison confirmed significant differences between treatments (data not shown).

Gene expression analyses: The total RNA was extracted, and cDNA strands were synthesized as described previously (Minaeva et al. 2017). Real-time quantitative reverse transcription PCR reactions were performed on a light cycler (CFX96, Bio-Rad, Hercules, USA) using SYBR Green I following a previously reported protocol (Zalutskaya et al. 2016). The primer sequences are listed in Table 1 Suppl. The relative gene expression ratios were normalized with a receptor of activated protein kinase $C$ (formerly termed $C B L P$ ) using the $\triangle \mathrm{Ct}$ and $\triangle \triangle \mathrm{Ct}$ methods (Livak and Schmittgen 2001). 
Measurement of NO: The NO content in cells was measured using 4-amino-5-methylamino-2'7'difluorofluorescein diacetate dye (Sigma-Aldrich) using a microplate reader CLARIOstar (BMG, Ortenberg, Germany) as described by Filina et al. (2019). The selective NO scavenger 2-phenyl-4,4,5,5-tetramethylimidazoline1-oxyl 3-oxide (cPTIO; Sigma-Aldrich) was used in a final concentration of $200 \mu \mathrm{M}$. Three technical replicates per condition were included on each plate, and each experiment was performed three times independently.

Statistics: The values are means \pm SEs of three biological replicates and three technical replicates. The differences between means were analyzed using the Student's $t$-test. Differences were considered statistically significant at $P<0.05$ and highly significant at $P<0.01$.

\section{Results}

To identify the potential impact of $\mathrm{NO}$ on proline accumulation, we used DEA-NONOate as NO donor (Floryszak-Wieczorek et al. 2006). The concentrations of DEA-NONOate used (50 and $100 \mu \mathrm{M}$ ) caused a significant increase in the fluorescence, showing the formation of NO (Fig. 2A). Notably, DEA-NONOate had no such effect after preincubation of cells with 2-phenyl-4,4,5,5tetramethylimidazoline-1-oxyl 3-oxide (cPTIO). Proline content increased progressively after addition of 50 or $100 \mu \mathrm{M}$ DEA-NONOate, reaching a maximum between 2 and $4 \mathrm{~h}$, where we measured 4.5- to 5-fold higher proline content than in untreated cells (Fig. $2 B$ ). When C. reinhardtii was exposed to $100 \mu \mathrm{M}$ DEA-NONOate, we observed a slightly larger accumulation of proline than in the presence of $50 \mu \mathrm{M}$ of $\mathrm{NO}$ donor. Therefore, $100 \mu \mathrm{M}$ of DEA-NONOate was chosen for further analysis.

Next, to demonstrate the function of NO in proline accumulation, we examined the abundance of transcripts for each gene in the glutamate and ornithine pathways (Fig. 1) in DEA-NONOate-treated versus untreated cells. Chlamydomonas reinhardtii genome contains two glutamyl kinase $(G G K)$ genes encoding $\gamma$-glutamyl kinase, the first enzyme of the proline biosynthetic pathway. We found that only $G G K 1 \mathrm{mRNA}$ was induced in response to DEANONOate treatment (Fig. 2C). In contrast, the transcript abundances of $G G K 2$ and PCR1 were down-regulated. Additionally, glutamate-5-semialdehyde dehydrogenase (GSD1) showed a 4.3-fold increase in its transcript abundance after $4 \mathrm{~h}$ of incubation (Fig. 2C). Proline may also be synthesized from ornithine by the ornithine- $\delta$ aminotransferase (OTA1) (Fig. 1), but OTA1 expression was downregulated by NO (Fig. 2C). The results also showed that the transcription of proline dehydrogenase 1, which catabolizes proline into P5C, declined significantly after $1 \mathrm{~h}$ (Fig. 2D), suggesting inhibition of proline catabolism.

We also tested the two genes of the arginine catabolism pathway that encode arginine deiminase $(A D I 1)$ and ornithine carbamoyltransferase (OTC1), two enzymes, producing ornithine from arginine (Fig. 1). Interestingly,
$A D I 1$ and OTC1 genes were induced after $30 \mathrm{~min}$ of exposure to NO donor (Fig. 2D). In C. reinhardtii, ornithine also can serve as a precursor for putrescine biosynthesis. Notably, ornithine decarboxylase 2 (ODC2), which converts ornithine into putrescine, was induced within 1 to $4 \mathrm{~h}$ of incubation with DEA-NONOate (Fig. $3 A$ ). Next, we asked the question whether the increased $O D C 2$ mRNA correlate with a change in putrescine content. Treatment of $C$. reinhardtii cells with NO donor resulted in a 2-fold increase in putrescine over the 4-h exposure period (Fig. 3B).

To further prove the role of NO in proline accumulation, free proline content and transcription of $G G K 1$ were compared in wild-type and THB2-amiRNA strains with reduced content of truncated hemoglobin 2 (Filina et al. 2019). Knockdown of THB2 results in increased NO production (Fig. 4A). Compared to the wild type (WT), proline content was increased in THB2-underexpressing strains (Fig. 4B). In addition, in amiRNA-THB2 strains, $G G K 1$ transcriptions were higher than in parental strain (Fig. 4B).

As $G G K 1$ and $O D C 2$ are NO-inducible genes (Fig. $2 C, 3 A$ ), we speculate that they may be upregulated during stresses, where NO plays a role, such as hypoxia and salt stress (Hemschemeier et al. 2013, Chen et al. 2016). Expression analysis of $G G K 1$ and $O D C 2$ showed that these genes were inducible by salt and hypoxia (Fig. 5A,C). However, there was a significant difference between the salt- and low oxygen-treated $C$. reinhardtii in GGK1 expressions, so that, $\mathrm{NaCl}$-exposed cells showed significantly more transcript abundance (with the highest induction of 2000-fold after $4 \mathrm{~h}$ ) than hypoxia-exposed cells (with the highest induction of 5-fold after $2 \mathrm{~h}$ ). In contrast, $O D C 2$ transcriptions were very similar under both stress conditions (Fig. 5A,C). Analyses of proline and putrescine content upon two stresses showed that salt treatment resulted in higher proline and putrescine content in cells in comparison with the hypoxia-exposed cells (Fig. 5B,D).

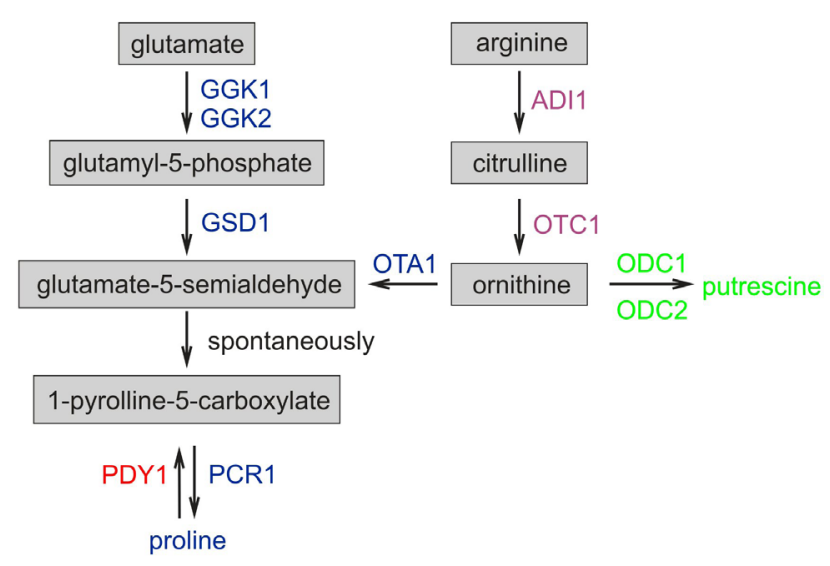

Fig. 1. Proline and putrescine biosynthesis in Chlamydomonas reinhardtii. Enzymes of proline and putrescine biosynthesis pathways are shown in blue and green, respectively. Red and purple colours refer to catabolic enzymes of proline and arginine, respectively. 


\section{Discussion}

Higher plants accumulate proline and putrescine as protective molecules in response to various environmental stresses (Szabados and Savouré 2010, Sengupta et al. 2016). In $C$. reinhardtii, proline and putrescine accumulation has been reported during salt stress (Mastrobuoni et al. 2012) and NO is involved the signaling pathways under these conditions (Chen et al. 2016). Until now, the effect of NO on proline and putrescine content in $C$. reinhardtii remains unclear. Here, we provide the first evidence that NO induced both proline and putrescine accumulation.

NO donor resulted in increased proline content, which was more abundant after exposure to $100 \mu \mathrm{M}$ compared with $50 \mu \mathrm{M}$ of DEA-NONOate, respectively (Fig. 2B). This might be explained by the fact that $100 \mu \mathrm{M}$ of DEANONOate produced higher NO amount than $50 \mu \mathrm{M}$ of NO donor (Fig. 2A). Since elevated NO content was detected in THB2-under-expressing cells (Filina et al. 2019), we used these strains in our experiments. Our analyses showed that under nonstress conditions THB2-knockdown strains maintained higher NO content than WT (Fig. 4A). In agreement with these data, THB2-amiRNA cells showed enhanced content of proline compared to the parental strain
(Fig. $4 B$ ). The content of proline and of intracellular NO in $T H B 2$-knockdown cells fell within the range normally seen in WT during treatment with $100 \mu \mathrm{M}$ of DEA-NONOate (Figs. 2 and 4). In other words, knockdown of THB2 induced some NO acculumation leading to an increase in proline content under nonstress conditions.

In land plants, intracellular proline accumulation is determined by its biosynthesis, catabolism, and transport (Verbruggen and Hermans 2008). C. reinhardtii is not able to transport external proline (Muños-Blanco et al. 1990). Importantly, a strong correlation between P5CS expression and the accumulation of proline has been shown in rice and Arabidopsis thaliana (Hein et al. 2003, Szekely et al. 2008). Therefore, transcription of key genes in proline metabolism of $C$. reinhardtii was tested in the presence of DEA-NONOate. Our real-time qPCR analysis of DEA-NONOate-treated $C$. reinhardtii cells indicated an increase in mRNA of $G G K 1$, an enzyme of glutamate biosynthetic pathway (Fig. 2C). The second NO-inducible gene, glutamate-5-semialdehyde dehydrogenase, showed a delayed upregulation. These results suggest that GGK1 might be the regulatory point controlling NO-dependent proline biosynthesis at the transcriptional level. Supporting evidence for this also comes from studies in THB2-

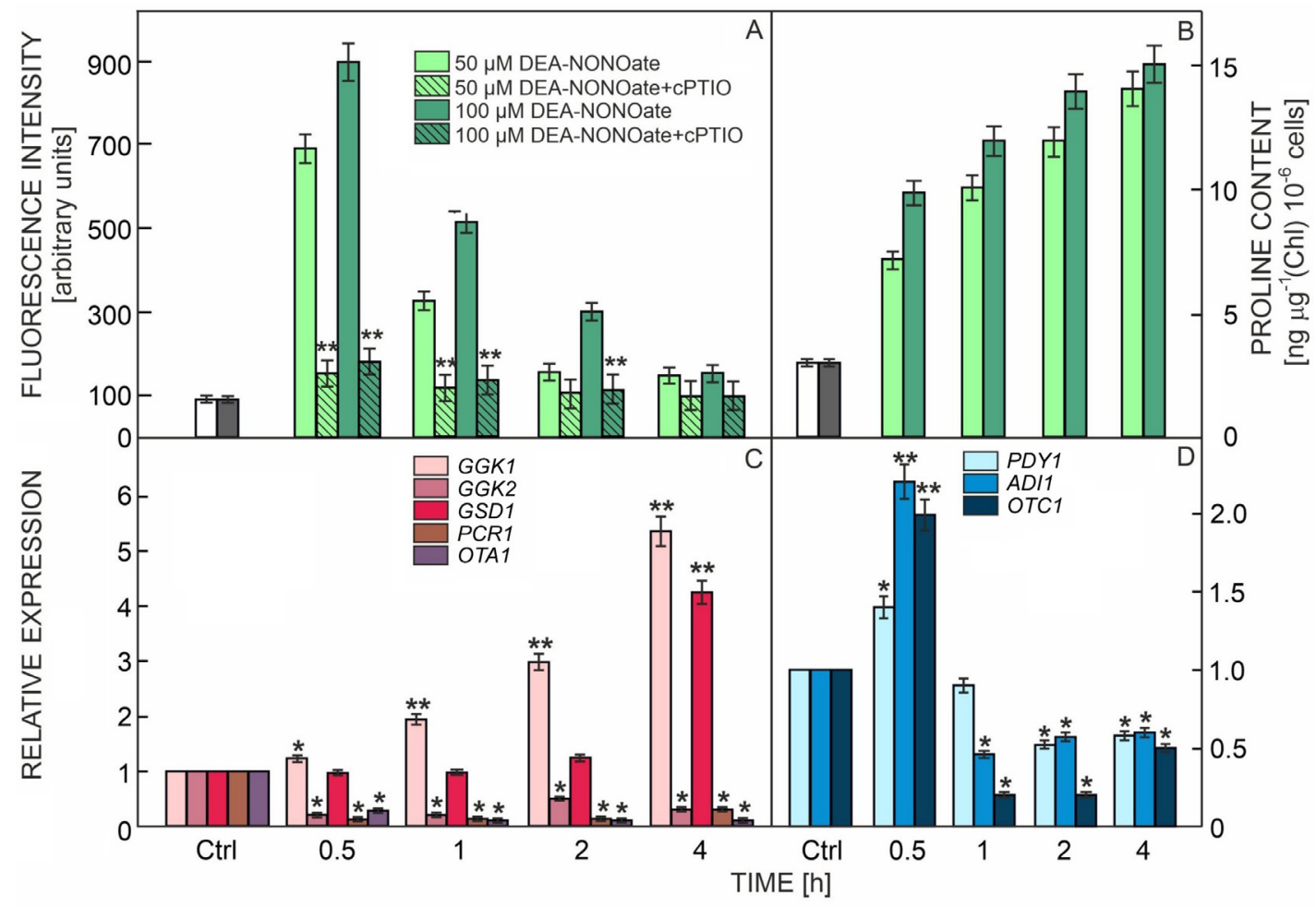

Fig. 2. Effects of 2-(N,N diethylamino)-diazenolate-2-oxide sodium salt (DEA-NONOate) on intracellular NO generation $(A)$, proline content $(B)$, relative expressions of genes of proline biosynthesis $(C)$ and proline and arginine catabolism $(D)$. $A$ - fluorescence intensity due to NO formation was determined using $1 \mu \mathrm{M}$ 4-amino-5-methylamino-2'7'-difluorofluorescein diacetate dye and expressed as arbitrary units per $1 \mu \mathrm{g}$ of chlorophyll in $10^{6}$ cells. Cell autofluorescence was subtracted from the total fluorescence obtained. The solution of $200 \mu \mathrm{M}$ 2-phenyl-4,4,5,5-tetramethylimidazoline-1-oxyl 3-oxide (cPTIO) was added 5 min before DEA-NONOate. $B$ - proline content is expressed as nanogrammes per $1 \mu \mathrm{g}$ of chlorophyll in $10^{6}$ cells. $C$ - relative expressions were determined in the presence of $100 \mu \mathrm{M}$ DEA-NONOate. Values are given as expressions relative to a house-keeping gene - receptor of activated protein kinase $C$, which had a value of 1 . Ctrl - control (non-treated cells). Means \pm SEs of three biological replicates and three technical replicates. Statistical analysis was performed by Student's $t$-test. In $(A) * *$ - significant differences between variants without and with cPTIO at $P<0.01$. In $(C, D) * * *$ - significant differences between non-treated and treated cells at $P \leq 0.05$ and 0.01 , respectively. 
underexpressing strains where enhanced endogenous NO resulted in higher $G G K 1$ mRNA abundances than in

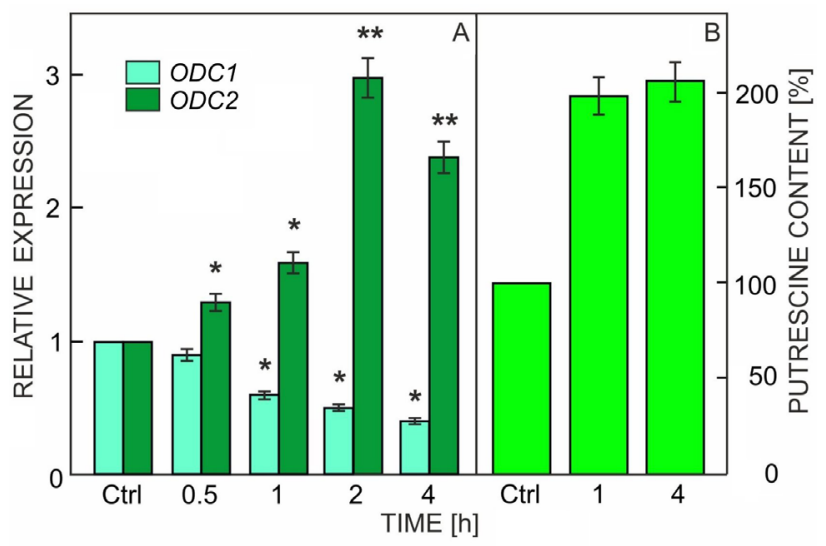

Fig. 3. Effects of 2-(N,N diethylamino)-diazenolate-2-oxide sodium salt (DEA-NONOate) on relative expressions of genes for putrescine biosynthesis $(A)$ and on putrescine content $(B)$. Relative gene expressions were determined as described in Fig. $2 C$. $B$ - putrescine amount in cells without added DEANONOate represents control (Ctrl, set to $100 \%$ ). Means \pm SEs of three biological replicates and three technical replicates. Statistical analysis was performed by Student's $t$-test. *,** significant differences between non-treated and treated cells at $P \leq 0.05$ and 0.01 , respectively.

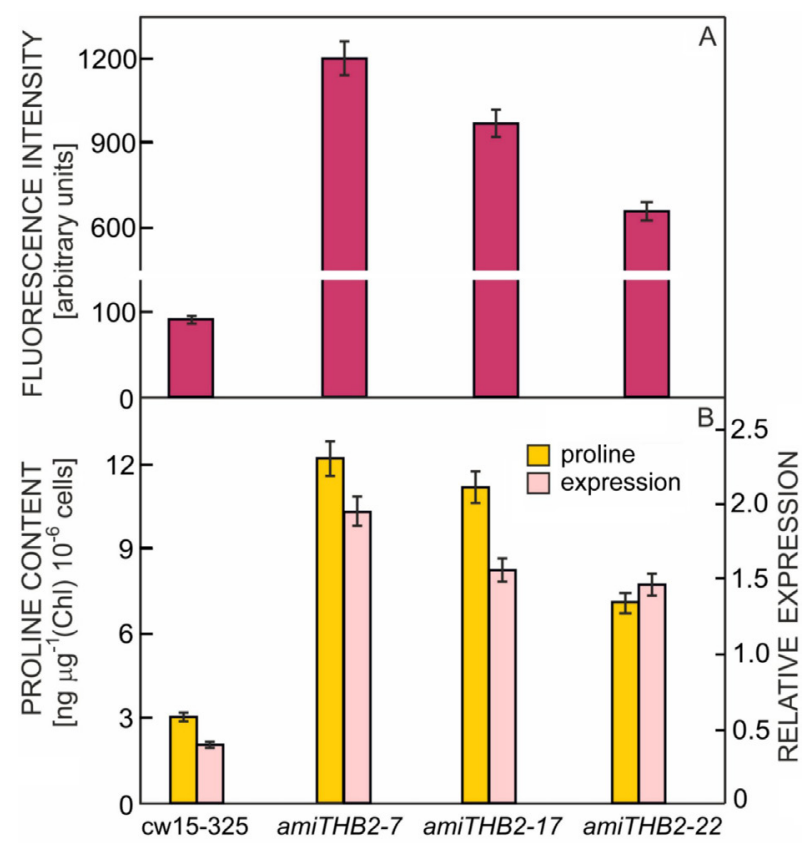

Fig. 4. Nitric oxide generation $(A)$ and proline content and glutamyl kinase 1 (GGKl) expression $(B)$ in parental and THB2-amiRNA strains of Chlamydomonas reinhardtii. Fluorescence intensity and proline content were expressed as described in Fig $2 A, B$. The relative $G G K 1$ expression was normalized to the expression of a receptor of activated protein kinase $C$ and calculated using $\Delta \mathrm{C}_{\mathrm{T}}$. Means \pm SEs of three biological replicates and three technical replicates. Statistical analysis was performed by Student's $t$-test. Differences were considered statistically significant at $P<0.01$.
WT (Fig. 4C). Notably, PCRl gene is repressed by NO (Fig. 2C), suggesting that like in higher plants (Filippou et al. 2013), $\Delta^{1}$-pyrroline-5-carboxylate reductase appeared no to be a rate-limiting factor in proline synthesis in this alga. We also found that the expression of proline dehydrogenase gene encoding a key-enzyme in proline catabolism is downregulated after NO-treatment (Fig. 2D). In $C$. reinhardtii, the mode of action of $\mathrm{NO}$ is poorly understood. The best studied NO-dependent pathway leads to the repression of genes involved in nitrate assimilation by ammonium and involves a soluble guanylate cyclase (De Montaigu et al. 2010, Sanz-Luque et al. 2016). The components of NO-based signaling pathway(s) that ate involved in induction of $G G K 1, O D C 2$, and inhibition of proline dehydrogenase 1 needs to be further elucidated.

In legume plants, the ornithine pathway (through enhanced transcription of $O A T$ encoding ornithine $\delta$-aminotransferase) may also contribute to the stressinduced proline accumulation (Armengaud et al. 2004). In $C$. reinhardtii, supplementation with DEA-NONOate decreased transcription of OTA1 (Fig. 2C), indicating that this pathway is rather repressed by NO. This suggests predominance of the glutamate pathway over the ornithine pathway.

Importantly, putrescine, which is essential metabolite of C. reinhardtii, is synthesized in the ornithine decarboxylase (ODC) pathway (Voigt et al. 2004), which after deimination of arginine to citrulline and decarboxylation to ornithine, yields putrescine by action of ODCs (Fig. 1). We hypothesized that in DEA-NONOate-treated cells, ornithine may be used as a precursor not for proline but for putrescine biosynthesis. Indeed, we demonstrated that in contrast to $A D I 1$ and $O T C 1, O D C 2$ gene is induced by NO (Fig. 3A). Additionally, NO-dependent increase in $O D C 2$ mRNA abundance accompanied by a 2-fold increase in putrescine content (Fig. 3B), suggesting that another protective molecule is also regulated by nitric oxide in C. reinhardtii. However, we cannot exclude the possibility that putrescine is accumulated, at least in part, due to other components. For example, the contribution of arginine decarboxylase to the formation of putrescine has been reported (Tassoni et al. 2018). Interestingly, in Medicago truncatula, exogenous NO controls putrescine and proline content via enzymatic and transcriptional modulation of proline and polyamine biosynthetic pathways (Filippou et al. 2013).

The observed NO-controlled accumulation of proline and putrescine suggest that both may come into the same stress(es) scene. Previously, it was shown that $C$. reinhardii produced enhanced content of proline and putrescine under salt stress (Mastrobuoni et al. 2012). Here, we clearly demonstrated that the increased conent of proline and putrescine was accompanied with the concurrent up-regulation of the $G G K 1$ and $O D C 2$ transcriptions, respectively (Fig. 5A,B). The induced expression of $G G K 1$ and $O D C 2$ genes to generate proline and putrescine in hypoxia is also documented in the present study (Fig. 5C,D). However, whether NO is essential for accumulation of proline and putrescine via up-regulation of the $G G K 1$ and $O D C 2$ genes under salt and hypoxic 

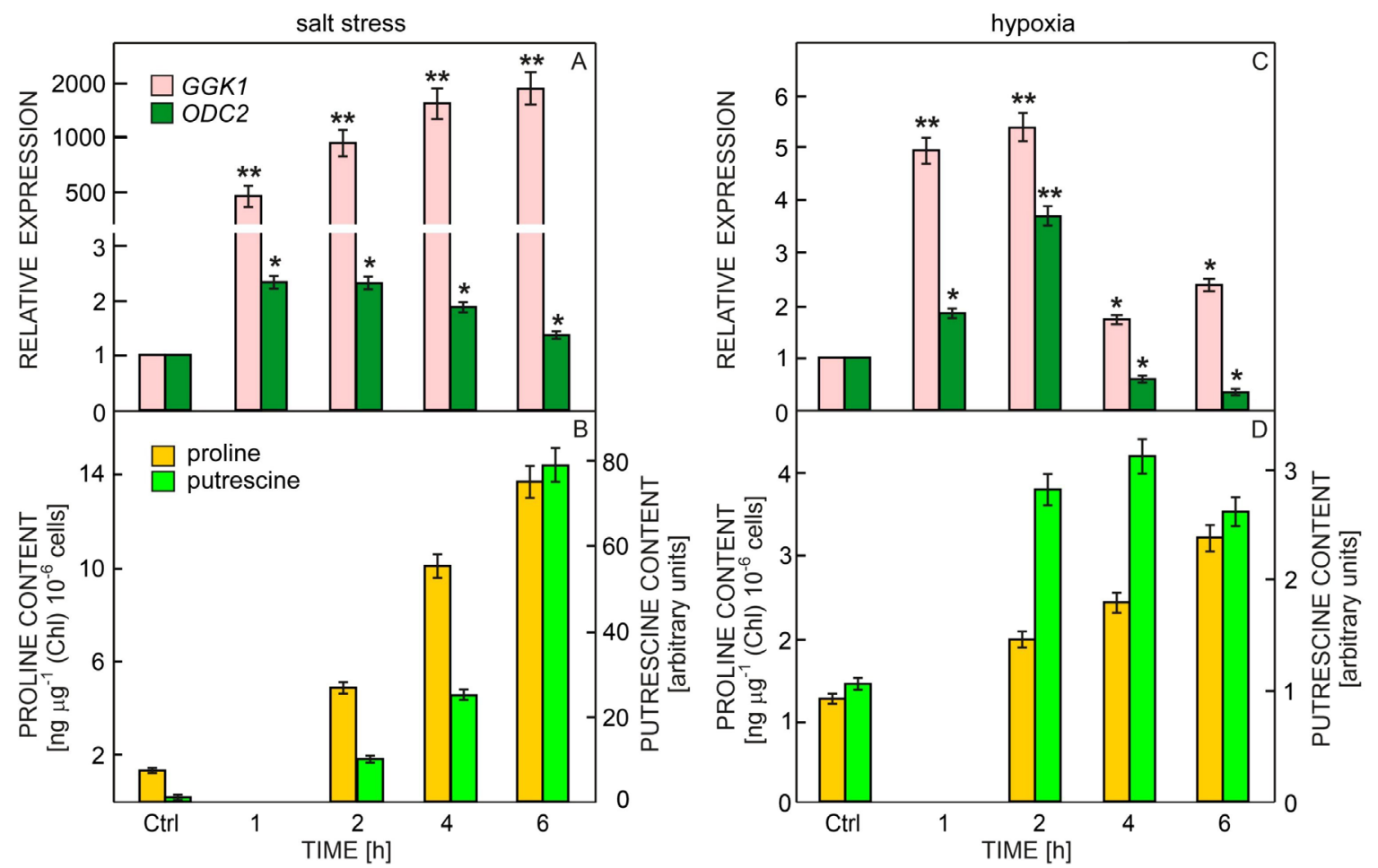

Fig. 5. Effects of salt stress and hypoxia on glutamyl kinase 1 (GGK1) and ornithine decarboxylase 2 (ODC2) transcript accumulations $(A, C)$, and proline and putrescine abundances $(B, D)$. Relative gene expressions and proline content were determined as described in Fig. 2B,C. Putrescine in non-stressed cells represent control (Ctrl, set to 1.0). Means \pm SEs of at least three biological replicates and three technical replicates. Statistical analysis was performed by Student's $t$-test. *** - significant differences between non-treated and treated cells at $P \leq 0.05$ and 0.01 , respectively.

stresses requires further investigation.

In conclusion, characterization of the NO-dependent biosynthesis of proline and putrescine expands our understanding of the regulatory aspects of NO signaling network in $C$. reinhardtii beyond previous findings (Hemschemeier et al. 2013, Wei et al. 2014, Chen et al. 2016, Minaeva et al. 2017, De Mia et al. 2019, Filina et al. 2019). The implication of NO in the control of the two protective molecules suggests the existence of a molecular mechanism that may coordinate different cellular responses under stress conditions.

\section{References}

Armengaud, P., Thiery, L., Buhot, N., Grenier-De March, G., Savouré, A.: Transcriptional regulation of proline biosynthesis in Medicago truncatula reveals developmental and environmental specific features. - Physiol. Plant. 120: 442-450, 2004.

Bates, L.S., Waldren, R.P., Teare, E.D.: Rapid determination of free proline for water stress studies. - Plant Soil 39: 205-207, 1973.

Chen, X., Tian, D., Kong, X., Chen, Q., Ef, A.A., Hu, X., Jia, A.: The role of nitric oxide signalling in response to salt stress in Chlamydomonas reinhardtii. - Planta 244: 651-669, 2016.

Chun, S.C., Paramasivan, M., Chandrasekaran, M.: Proline accumulation influenced by osmotic stress in arbuscular mycorrhizal symbiotic plants. - Front. Microbiol. 9: 2525, 2018.
Commichau, F.M., Gunka, K., Landmann, J.J., Stülke, J.: Glutamate metabolism in Bacillus subtilis: gene expression and enzyme activities evolved to avoid futile cycles and to allow rapid responses to perturbations of the system. - J. Bacteriol. 190: 3557-3564, 2008.

Corpas, F.J., Barroso, J.B.: Nitric oxide from a "green" perspective. - Nitric Oxide 45: 15-19, 2015.

Corpas, F.J., Leterrier, M., Valderrama, R., Airaki, M., Chaki, M., Palma, J.M., Barroso, J.B.: Nitric oxide imbalance provokes a nitrosative response in plants under abiotic stress. - Plant Sci. 181: 604-611, 2011.

Dar, M.I., Naikoo, M.I., Rehman, F., Naushin, F., Khan, F.A.: Proline accumulation in plants: roles in stress tolerance and plant development. - In: Iqbal, N., Nazar, R., Khan, N.A. (ed.): Osmolytes and Plants Acclimation to Changing Environment: Emerging Omics Technologies. Pp. 155-166. Springer, New Delhi 2016.

De Mia, M., Lemaire, S.D., Choquet, Y., Wollman, F.A.: Nitric oxide remodels the photosynthetic apparatus upon S-starvation in Chlamydomonas reinhardtii. - Plant Physiol. 179: 718-731, 2019.

De Montaigu, A., Sanz-Luque, E., Macias, M., Galvan, A., Fernandez, E.: Transcriptional regulation of CDP1 and CYG56 is required for proper $\mathrm{NH}_{4}{ }^{+}$sensing in Chlamydomonas. - $\mathrm{J}$. exp. Bot. 62:1425-1437, 2011.

Domingos, P., Prado, A.M., Wong, A., Gehring, C., Feijo, J.A.: Nitric oxide: a multitasked signaling gas in plants. - Mol. Plants 8: 506-520, 2015.

Farnese, F.S., Menezes-Silva, P.E., Gusman, G.S., Oliveira, J.A.: When bad guys become good ones: the key role of reactive oxygen species and nitric oxide in the plant responses to 
abiotic stress. - Front. Plant Sci. 7: 471, 2016.

Filina, V., Grinko, A., Ermilova, E.: Truncated hemoglobins 1 and 2 are implicated in the modulation of phosphorus deficiencyinduced nitric oxide levels in Chlamydomonas. - Cells 8: 947, 2019.

Filippou, P., Antoniou, C., Fotopoulos, V.: The nitric oxide donor sodium nitroprusside regulates polyamine and proline metabolism in leaves of Medicago truncatula plants. - Free Rad. Biol. Med. 56: 172-183, 2013.

Fichman,Y., Gerdes, S., Kovács, S., Szabados,L., Zilberstein, Z., Csonka, L.: Evolution of proline biosynthesis: enzymology, bioinformatics, genetics, and transcriptional regulation. Biol. Rev. 90: 1065-1099, 2015.

Floryszak-Wieczorek, J., Milczarek, G., Arasimowicz, M., Ciszewski, A.: Do nitric oxide donors mimic endogenous NO-related response in plants. - Planta 224: 1363-1372, 2006.

Hein, D.T., Jacobs, M., Angenon, G., Hermans, C., Thu, T.T., Son, L.V., Roosens, N.H.: Proline accumulation and $\Delta 1$ pyrroline-5-carboxylate synthetase gene properties in three rice cultivars differing in salinity and drought tolerance. Plant Sci. 165: 1059-1068, 2003.

Hemschemeier, A., Düner, M., Casero, D., Merchant, S.S., Winkler, M., Happe, T.: Hypoxic survival requires a 2-on-2 hemoglobin in a process involving nitric oxide. - Proc. nat. Acad. Sci. USA 110: 10854-10859, 2013.

Hoffmann, T., Bremer, E.: Management of osmotic stress by Bacillus subtilis: genetics and physiology. - In: De Bruijn, F.J. (ed.): Stress and Environmental Regulation o Gene Expression and Adaptation in Bacteria. Vol. 1. Pp. 657-676. Wiley-Blackwell Publishers, Hoboken 2016.

Johnsen, L., Skou, P., Khakimov, B., Bro, R.: Gas chromatography - mass spectrometry data processing made easy. - J. Chromatogr. A. 1503: 57-64, 2017.

Livak, K.J., Schmittgen, T.D.: Analysis of relative gene expression data using real-time quantitative PCR and the $2^{-\Delta C T}$ method. - Methods 25: 402-408, 2001.

Mastrobuoni, G., Irgang, S., Pietzke, M., Assmus, H.E., Wenzel, M., Schulze, W.X., Kempa, S.: Proteome dynamics and early salt stress response of the photosynthetic organism Chlamydomonas reinhardtii. - BMC Genomics 13: 215, 2012.

Milani, M., Pesce, A., Ouellet, H., Guertin, M., Bolognesi, M.: Truncated hemoglobins and nitric oxide action. - IUBMB Life 55: 623-627, 2003.

Minaeva, E., Zalutskaya, Z., Filina, V., Ermilova, E.: Truncated hemoglobin 1 is a new player in Chlamydomonas reinhardtii acclimation to sulfur deprivation. - PLoS ONE 12: e0186851, 2017.

Miyoshi, K., Matsuura, H., Kumon, T., Matsuda, K., Yoshikawa, T., Bamba, T., Harada, K., Hirata, K.: Isolation and partial characterization of the Chlamydomonas reinhardtii $\gamma$-glutamyl kinase. - J. Biosci. Bioeng. 112: 75-78, 2011.

Muños-Blanco,J.,Hidalgo-Martinez,J.,Cárdenas, J.:Extracellular deamination of L-amino acids by Chlamydomonas reinhardtii cells. - Planta 182: 194-198, 1990.

Mur, L.A., Prats, E., Pierre, S., Hall, M.A., Hebelstrup, K.H.: Integrating nitric oxide into salicylic acid and jasmonic acid/ ethylene plant defense pathways. - Front. Plant Sci. 4: 215, 2013.

Ostroukhova, M., Zalutskaya, Z., Ermilova, E.: New insights into AOX2 transcriptional regulation in Chlamydomonas reinhardtii. - Eur. J. Protistol. 58: 1-8, 2017.

Puzanskiy, R., Tarakhovskaya, E., Shavarda, A., Shishova, M.: Metabolomic and physiological changes of Chlamydomonas reinhardtii (Chlorophyceae, Chlorophyta) during batch culture development. - J. appl. Phycol. 30: 803-818, 2017.
Sanz-Luque, E., Ocaña-Calahorro, F., de Montaigu, A., ChamizoAmpudia, A., Llamas, Á., Galván, A., Fernández, E.: THB 1, a truncated hemoglobin, modulates nitric oxide levels and nitrate reductase activity. - Plant J. 81: 467-479, 2015.

Sanz-Luque, E., Ocaña-Calahorro, F., Galván, A., Fernández, E., De Montaigu, A.: Characterization of a mutant deficient for ammonium and nitric oxide signalling in the model system Chlamydomonas reinhardtii. - PloS ONE 11: e0155128, 2016.

Sengupta, A., Chakraborty, M., Saha, J., Gupta, B., Gupta, K.: Polyamines: Osmoprotectants in plant abiotic stress adaptation. - In: Iqbal, N., Nazar, R., Khan, N.A. (ed.): Osmolytes and plants acclimation to changing environment: emerging omics technologies. Pp. 97-127. Springer, New Delhi 2016.

Sharma, S., Villamor, J.G., Verslues, P.E.: Essential role of tissuespecific proline synthesis and catabolism in growth and redox balance at low water potential. - Plant Physiol. 157: 292-304, 2011.

Signorelli, S.: The fermentation analogy: a point of view for understanding the intriguing role of proline accumulation in stressed plants. - Front. Plant Sci. 7: 1339, 2016.

Slocum, R.D.: Tissue and subcellular localization of polyamines and enzymes of polyamine metabolism. - In: Slocum, R.D., Flores, H.E. (ed.): Biochemistry and Physiology of Polyamines in Plants. Pp. 23-40. CRC Press, Boca Raton 1991.

Szabados, L., Savouré, A.: Proline: a multifunctional amino acid. - Trends Plant Sci. 15: 89-97, 2010.

Székely, G., Abrahám, E., Cséplo, A., Rigó, G., Zsigmond, L., Csiszár, J., Ayaydin, F., Strizhov, N., Jásik, J., Schmelzer, E., Koncz, C., Szabados, L.: Duplicated P5CS genes of Arabidopsis play distinct roles in stress regulation and developmental control of proline biosynthesis. - Plant J. 53: $11-28,2008$.

Szepesi, A., Szőllősi, R.: Mechanism of proline biosynthesis and role of proline metabolism enzymes under environmental stress in plants. - In: Ahmad, P., Ahanger, M.A., Pratap, V., Tripathi, S.D.K., Alam, P., Alyemeni, M.N. (ed.): Plant Metabolites and Regulation under Environmental Stress. Pp. 337-353. Elsevier, San Diego 2018.

Tassoni, A., Awad, N., Griffiths, G.: Effect of ornithine decarboxylase and norspermidine in modulating cell division in the green alga Chlamydomonas reinhardtii. - Plant Physiol. Biochem. 123: 125-131, 2018.

Verbruggen, N., Hermans, C.: Proline accumulation in plants: a review. - Amino Acids 35: 753-759, 2008.

Voigt, J., Deinert, B., Bohley, P.: Subcellular localization and light-dark control of ornithine decarboxylase in the unicellular green alga Chlamydomonas reinhardtii. - Physiol. Plant. 108: 353-360, 2000.

Voigt, J., Fausel, M., Bohley, P., Adam, K.H., Marquardt, O.: Structure and expression of the ornithine decarboxylase gene of Chlamydomonas reinhardtii. - Microbiol. Res. 159: 403417, 2004.

Wei, L., Derrien, B., Gautier, A., Houille-Vernes, L., Boulouis, A., Saint-Marcoux, D., Malnoe, A., Rappaport, F., De Vitry, C., Vallon, O., Choquet, Y., Wollman, F.A.: Nitric oxide-triggered remodeling of chloroplast bioenergetics and thylakoid proteins upon nitrogen starvation in Chlamydomonas reinhardtii. Plant Cell 26: 353-372, 2014.

Wendehenne, D., Hancock, J.: New frontiers in nitric oxide biology in plant. - Plant Sci. 181: 507-620, 2011.

Zalutskaya, Z., Ostroukhova, M., Ermilova, E.: The Chlamydomonas alternative oxidase 1 is regulated by cadmium stress: new insights into control of expression. Environ. exp. Bot. 130: 133-140, 2016. 